\title{
MOLECULAR BASIS OF THE VARIATION EXHIBITED BY AVIAN INFECTIOUS
}

BRONCHITIS CORONAVIRUS (IBV)

D. Cavanagh, P. Davis, J. Cook and D. Li

AFRC Institute for Animal Health, Houghton Laboratory

Houghton, Huntingdon, Cambridgeshire, PE17 2DA, UK

\section{ABSTRACT}

IBV serotype-specific, virus neutralising (VN) antibody is induced by the S1 subunit of the spike (S) glycoprotein. A Portuguese isolate (Port/322/85) is considered to be an in vivo recombinant. VN tests showed that it belongs to the Massachussetts (Mass) serotype. Correspondingly, the sequence of $\mathrm{S} 1$ and S2 was extremely similar to that of older Mass isolates. However, the matrix (M) glycoprotein and upstream gene sequences were atypical of these Mass strains and more closely resembled other serotypes isolated in Europe. The recombination event had occurred within the sequence between the $M$ and $S$ genes (corresponding to mRNA 3) or near the $3^{\prime}$ terminus of the $\mathrm{S} 2$ gene.

\section{INTRODUCTION}

It has been known for decades that IBV exhibits extensive antigenic variation. On the basis of $\mathrm{VN}$ tests isolates have been assigned to serotypes, the number of which continues to increase ${ }^{1,2}$. Serotypespecific VN antibody is induced by $\mathrm{S} 1$, the amino-(N)-terminal half of the spike (S) glycoprotein ${ }^{3-6}$. Moreover, amino acid differences between isolates are concentrated within the $\mathrm{N}$-terminal $25 \%$ of S1 residues ${ }^{6-8}$ and some epitopes for VN antibody are situated in this region ${ }^{6}$ (Fig.1).

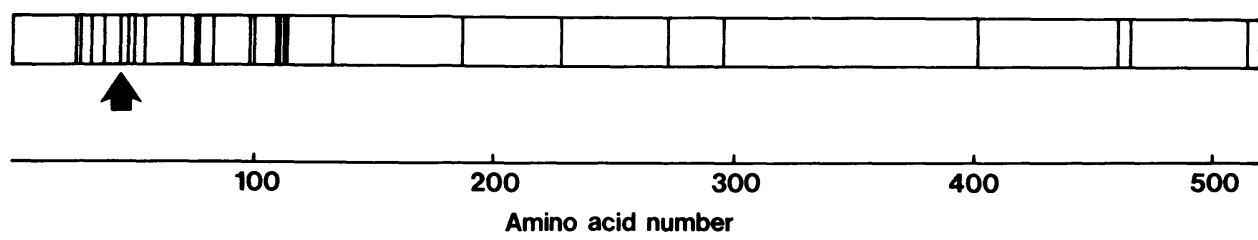

Fig.1. Amino acid variation within S1 of 8 Mass serotype strains (from reference 6 ). Vertical lines indicate where at least one of seven Mass strains differed in sequence from the M41 strain. The arrow shows the position of an amino acid substitution in a mutant of M41 which resisted neutralization by a monoclonal antibody. ${ }^{6}$ 
In this communication we present evidence that a strain of the Massachusetts serotype is actually a recombinant.

METHODS

Isolate Portugal/322/85 was received in 1985 and was serotyped by virus neutralization (VN) tests in chicken tracheal organ cultures. IBV RNA was produced and sequenced essentially as described previously ${ }^{\circ}$.

\section{RESULTS}

Strains of the Mass serotype have always been a major cause of severe outbreaks of IB and the most widely used vaccines are of the Mass serotype. Our analysis of a number of vaccine and field strains, all of the Mass serotype but isolated over a four decade period, showed that S1 had been strongly conserved (S2 was not sequenced) ${ }^{6}$ (Fig.1). Taken as a group, the 7 strains analysed varied from the M41 strain, at only $6 \%$ of amino acids. The $3 c$ ORF and $M$ gene of the vaccinal strains had also been highly conserved ${ }^{9}$.

Isolate Port/322/85 was neutralised by antisera to several Mass strains including $\mathrm{M} 41$ and $\mathrm{H} 120$ and was considered to be of the Mass serotype. Sequencing of S1 gave data which correlated with the serological analysis. Thus the S1 protein of Port/322/85 shared $94.9 \%$ homology with that of M41 (Fig.2). Several of the amino acids possessed by Port/322/85 and different from $M 41$ were identical to those in the vaccine strain H120, the field strain HVI-140 (Fig.2) and the other Mass strains previously sequenced ${ }^{6}$. However, the $3 c$ ORF and $M$ gene sequence of Port/322/85 was not at all like that of these other Mass strains (Fig.3). Rather, it more closely resembled the sequences of UK/6/82 and $\mathrm{UK} / 200 / 83^{9}$, both in terms of deletions and the possession of specific nucleotides. We then sequenced the S2 part of the $\mathrm{S}$ gene. There was $97.4 \%$ amino acid homology with S2 of $M 41$ (we determined $87 \%$ of the S2 sequence of Port/322/85). We were not able to determine the carboxy-(C-)-terminal amino acids because of the complete failure of the oligonucleotide used to prime the reverse transcription of this part of the gene. The oligonucleotide was complementary to IBV-M4I sequence a few nucleotides downstream from the C-terminus of $\mathrm{S} 2$, in the $3 \mathrm{a}$ ORF.

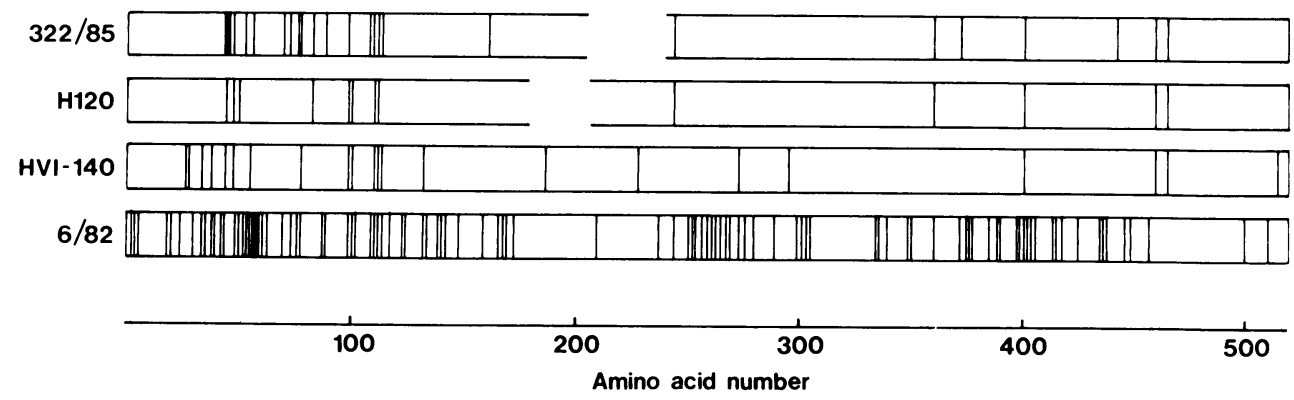

Fig.2. Comparison of the $\mathrm{S} 1$ amino acid sequence of Mass serotype strains Port/322/85, H120 and HVI-140 and the non-Mass strain UK/6/82 with that of the M41 strain. Each vertical line represents an amino acid difference. 
(a) UGGUAGAAAACUUAACAAUCCGGAAUUAGAAGCAGUUAUUGUCAACGAGUUUCCUAAGAACGGUUGG AAUAAUAAAAAUCCAGCAAAUUUUCAAG $\mathrm{MM} /$ Bronch $\mathrm{H} 52 / \mathrm{H} 12 \mathrm{O}$

Ibvax

$\begin{array}{ll}322 / 85 & A \\ U K / 200 / 83 C & A\end{array}$

$\mathrm{UK} / 6 / 82 \quad$ C $\quad A$

(b) AUGUCCAACGAGACAAAUUGUACUCUUGACUUUGAACAGUCAGUUGAGCUUUUUAAAGAGUAUAAU

$\mathrm{Mm} /$ Bronch

H52/H12O

Ibvax

$322 / 85 \quad * * * * * * * * * A$

UK $/ 200 / 83 * * * *$ UGACAGA

UK/6/82 GAU ACC C

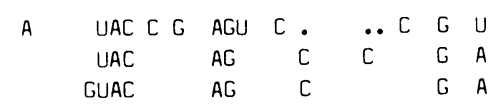

(c)

M41 UUAUUUAUAACUGCAUUCUUGUUGUUCUUAACCAUAAUACUUCAGUAUGGCUAUGCAACAAGAAGUAAC

$\mathrm{Mm} /$ Bronch

H52/H12O

Ibvax

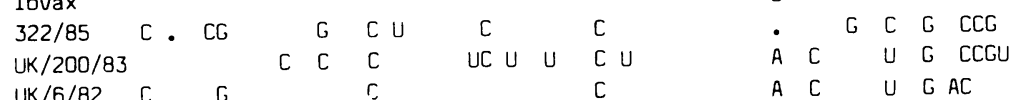

$U K / 6 / 82 \quad C \quad G \quad C D \quad C \quad A \quad C \quad \cup \quad G A C$

(d)

M41,MM,Bronch, MSNETNCTLDFEQSVELFKEYNLF I TAFLLFLTIILQYGYATRSK

H52, H120, Ibvax

\begin{tabular}{|c|c|c|c|c|c|}
\hline $322 / 85$ & $* * \# M$ & & VA. . D & v & L \\
\hline UK/200/83 & \#\#MTE & $T$ & $A Q$ & & L \\
\hline UK/6/82 & $D T$ & GT & $A Q$ & v & L \\
\hline
\end{tabular}

Fig.3. M gene sequences. Only those nucleotides which differ from those of M41 are shown; unidentified nucleotides are marked with a dot (.). (a) sequences of the $3^{\prime}$ end of the $3 \mathrm{c} 0 \mathrm{RF}$; (b) sequence coding for the exposed part of $M$; (c) sequence for the first membrane-embedded part of $M$. Nucleotides absent from some sequences are indicated by asterisks *; (d) comparison of the amino acid sequences of the $M$ protein corresponding to nucleotide sequences in (b) and (c).

The striking similarity of the $S 1$ genes of Port/322/85 and M41 and equally striking dissimilarity of their $3 \mathrm{c}$ ORF and $M$ sequences indicates that Port/322/85 is a recombinant. The recombinantion event would have occurred either within the gene 3 ORFs or, given the failure of the above-mentioned oligonucleotide, near the C-terminus of $\mathrm{S} 2$.

\section{DISCUSSION}

The results presented herein and the recent findings of Kusters et al. ${ }^{8}$ strongly support the view that some IBV isolates are naturaliy occurring recombinants. Given the high frequency of recombination which occurred during experimental mixed infections with murine hepatitis virus strains ${ }^{10}$ it seems likely that many IBV strains are recombinant at one or more loci.

Strains of the Mass serotype continue to be isolated, 50 years after the first Mass isolate was made. Given the widespread use of Mass serotype vaccines - some countries, including the UK, permit only live vaccines of this serotype - it might be assumed that a Mass isolate is a re-isolate of a vaccine strain. Sequencing of $\mathrm{S} 1$ can indicate if a

Mass isolate is a vaccine strain, a mutant of a vaccine or a nonvaccine related Mass field strain (which from the data reported herein, some 
isolates clearly are). However, given the phenomenon of recombination, such data can be no more than a tentative indication of the identity of an isolate. Sequencing of the $M$ gene improves the situation but this still leaves unexamined more than 20000 nucleotides, among which there may have been point mutations, deletions, insertions and/or recombination events!

\section{ACKNOWLEDGEMENT}

We wish to thank Miss Carolyn Payne and Mrs Marjorie Ellis for technical assistance.

\section{REFERENCES}

1. Cook, J.K.A. The classification of new serotypes of infectious bronchitis virus isolated from poultry flocks in Britain between 1981 and 1983. Avian Path. 13: 733 (1984).

2. Cook, J.K.A. and Huggins, M.B. Newly isolated serotypes of infectious bronchitis virus: their role in disease. Avian Path. 15: 129 (1986).

3. Cavanagh, D., Darbyshire, J.H., Davis, P. and Peters, R.W. Induction of humoral neutralising and haemagglutination-inhibiting antibody by the spike protein of avian infectious bronchitis virus. Avian Path. 13: 573 (1984).

4. Mockett, A.P., Cavanagh, D and Brown, T.D.K. Monoclonal antibodies to the S1 spike and membrane proteins of avian infectious bronchitis coronavirus strain Massachusetts M41. J. gen. Virol. 65: 2281 (1984).

5. Cavanagh, D., Davis, P.J., Darbyshire, J.H. and Peters, R.W. Coronavirus IBV: Virus retaining spike glycopolypeptide S2 but not S1 is unable to induce virus-neutralizing or haemagglutinationinhibiting antibody, or induce chicken tracheal protection. J. gen. Virol. 67: 1435 (1986).

6. Cavanagh, D., Davis, P.J. and Mockett, A.P.A. Amino acids within hypervariable region 1 of avian coronavirus IBV (Massachusetts serotype) spike glycoprotein are associated with neutralization epitopes. Virus Res. 11: 141 (1988).

7. Binns, M.M. Boursnell, M.E.G., Tomley, F.M. and Brown, T.D.K. Comparison of the spike precursor sequences of coronavirus IBV strains $M 41$ and $6 / 82$ with that of IBV Beaudette. J. gen. Virol. 67: 2825 (1986).

8. Kusters, J.G., Niesters, H.G.M., Lenstra, J.A., Horzinek, M.C. and van der Zeijst B.A.M. Phylogeny of antigenic variants of avian coronavirus IBV. Virology, 169: 217 (1989).

9. Cavanagh, D., and Davis, P.J. Evolution of avian coronavirus IBV: sequence of the matrix glycoprotein gene and intergenic region of several serotypes. J.gen.Virol. 69: 621 (1988).

10. Keck, J.G., Matsushima, G.K., Makino, S., Fleming, J.0., Vannier, D.M., Stohlman, S.A. and Lai, M.M.C. In vivo RNA-RNA recombination of coronaviruses in mouse brain. J. Virol. 62: 1810 (1988). 